\title{
Periodic boundary value problems for second-order impulsive integro-differential equations with integral jump conditions
}

\author{
Chatthai Thaiprayoon ${ }^{1,2^{*}}$, Decha Samana ${ }^{1,2}$ and Jessada Tariboon ${ }^{2,3}$
}

\author{
"Correspondence: \\ tik_kengjang@hotmail.com \\ ${ }^{1}$ Department of Mathematics, \\ Faculty of Science, King Mongkut's \\ Institute of Technology Ladkrabang, \\ Bangkok, 10520, Thailand \\ ${ }^{2}$ Centre of Excellence in \\ Mathematics, CHE, Sri Ayutthaya \\ Road, Bangkok, 10400, Thailand \\ Full list of author information is \\ available at the end of the article
}

\begin{abstract}
This paper is concerned with the existence of extremal solutions of periodic boundary value problems for second-order impulsive integro-differential equations with integral jump conditions. We introduce a new definition of lower and upper solutions with integral jump conditions and prove some new maximum principles. The method of lower and upper solutions and the monotone iterative technique are used.
\end{abstract}

MSC: 34B37; 34K10; 34K45

Keywords: impulsive integro-differential equation; lower and upper solutions; periodic boundary value problem; monotone iterative technique

\section{Introduction}

Differential equations which have impulse effects describe many evolution processes that abruptly change their state at a certain moment. In recent years, impulsive differential equations have become more important tools in some mathematical models of real processes and phenomena studied in physics, biotechnology, chemical technology, population dynamics and economics; see [1-5]. Many papers have been published about existence analysis of periodic boundary value problems of first and second order for impulsive ordinary or functional or integro-differential equations. We refer the readers to the papers [6-29]. More recent works on existence results of impulsive problems with integral boundary conditions can be found in [30-35] and the reference therein. This literature has lead to significant development of a general theory for impulsive differential equations.

The monotone iterative technique coupled with the method of upper and lower solutions has been used to study the existence of extremal solutions of periodic boundary value problems for second-order impulsive equations; see, for example, [36-41]. This method has been also used to study abstract nonlinear problems; see [42]. However, in most of these papers concerned with applications of the monotone iterative technique to secondorder periodic boundary value problems with impulses, the authors assume that the jump conditions at impulse point $t_{k}$ of solution values and the derivative of solution values depend on the left-hand limits of solutions or the slope of solutions themselves, such as $\Delta x\left(t_{k}\right)=I_{k}\left(x\left(t_{k}^{-}\right)\right), \Delta x\left(t_{k}\right)=I_{k}\left(x^{\prime}\left(t_{k}^{-}\right)\right), \Delta x^{\prime}\left(t_{k}\right)=I_{k}\left(x\left(t_{k}^{-}\right)\right), \Delta x^{\prime}\left(t_{k}\right)=I_{k}\left(x^{\prime}\left(t_{k}^{-}\right)\right)$. 
In this paper, we consider the periodic boundary value problem for second-order impulsive integro-differential equation (PBVP) with integral jump conditions:

$$
\left\{\begin{array}{l}
x^{\prime \prime}(t)=f(t, x(t),(K x)(t),(S x)(t)), \quad t \in J=[0, T], t \neq t_{k}, \\
\Delta x\left(t_{k}\right)=I_{k}\left(\int_{t_{k}-\delta_{k}}^{t_{k}-\varepsilon_{k}} x^{\prime}(s) d s\right), \quad k=1,2, \ldots, m, \\
\Delta x^{\prime}\left(t_{k}\right)=I_{k}^{\prime \prime}\left(\int_{t_{k}-\tau_{k}}^{t_{k}-\sigma_{k}} x(s) d s\right), \quad k=1,2, \ldots, m, \\
x(0)=x(T), \quad x^{\prime}(0)=x^{\prime}(T),
\end{array}\right.
$$

where $0=t_{0}<t_{1}<t_{2}<\cdots<t_{k}<\cdots<t_{m}<t_{m+1}=T, f: J \times R^{3} \rightarrow R$ is continuous everywhere except at $\left\{t_{k}\right\} \times R^{3}, f\left(t_{k}^{+}, x, y, z\right), f\left(t_{k}^{-}, x, y, z\right)$ exist, $f\left(t_{k}^{-}, x, y, z\right)=f\left(t_{k}, x, y, z\right), I_{k} \in$ $C(R, R), I_{k}^{*} \in C(R, R), \Delta x\left(t_{k}\right)=x\left(t_{k}^{+}\right)-x\left(t_{k}^{-}\right), \Delta x^{\prime}\left(t_{k}\right)=x^{\prime}\left(t_{k}^{+}\right)-x^{\prime}\left(t_{k}^{-}\right), 0 \leq \varepsilon_{k} \leq \delta_{k} \leq t_{k}-t_{k-1}$, $0 \leq \sigma_{k} \leq \tau_{k} \leq t_{k}-t_{k-1}, k=1,2, \ldots, m$,

$$
(K x)(t)=\int_{0}^{t} k(t, s) x(s) d s, \quad(S x)(t)=\int_{0}^{T} h(t, s) x(s) d s,
$$

$k(t, s) \in C\left(D, R^{+}\right), h(t, s) \in C\left(J \times J, R^{+}\right), D=\left\{(t, s) \in R^{2}, 0 \leq s \leq t \leq T\right\}, R^{+}=[0,+\infty), k_{0}=$ $\max \{k(t, s):(t, s) \in D\}, h_{0}=\max \{h(t, s):(t, s) \in J \times J\}$.

In $[43,44]$, the authors discussed some kinds of first-order impulsive problems with the integral jump condition

$$
\Delta x\left(t_{k}\right)=I_{k}\left(\int_{t_{k}-\tau_{k}}^{t_{k}} x(s) d s-\int_{t_{k-1}}^{t_{k-1}+\sigma_{k-1}} x(s) d s\right)
$$

where $0<\sigma_{k-1} \leq\left(t_{k}-t_{k-1}\right) / 2,0 \leq \tau_{k-1} \leq\left(t_{k}-t_{k-1}\right) / 2, k=1,2, \ldots, m$. We note that the jump condition (1.2) depends on functionals of path history before impulse points $t_{k}$ and after the past impulse points $t_{k-1}$. The aim of our research is to deal with the integral jump conditions

$$
\Delta x\left(t_{k}\right)=I_{k}\left(\int_{t_{k}-\delta_{k}}^{t_{k}-\varepsilon_{k}} x^{\prime}(s) d s\right), \quad \Delta x^{\prime}\left(t_{k}\right)=I_{k}^{\prime \prime}\left(\int_{t_{k}-\tau_{k}}^{t_{k}-\sigma_{k}} x(s) d s\right),
$$

where $0 \leq \varepsilon_{k} \leq \delta_{k} \leq t_{k}-t_{k-1}, 0 \leq \sigma_{k} \leq \tau_{k} \leq t_{k}-t_{k-1}, k=1,2, \ldots, m$. The integral jump condition (1.3) means that a sudden change of solution values and the derivative of solution values at impulse point $t_{k}$ depend on the area under the curves of $x^{\prime}(t)$ and $x(t)$ between $t=t_{k}-\delta_{k}$ to $t=t_{k}-\varepsilon_{k}$ and $t=t_{k}-\tau_{k}$ to $t=t_{k}-\sigma_{k}$, respectively. It should be noticed that the impulsive effects of PBVP (1.1) have memory of the past states.

This paper is organized as follows. Firstly, we introduce a new concept of lower and upper solutions. After that, we establish some new comparison principles and discuss the existence and uniqueness of the solutions for second-order impulsive integro-differential equations with integral jump conditions. By using the method of upper and lower solutions and the monotone iterative technique, we obtain the existence of an extreme solution of PBVP (1.1). Finally, we give an example to illustrate the obtained results.

\section{Preliminaries}

Let $J^{-}=J \backslash\left\{t_{1}, t_{2}, \ldots, t_{m}\right\}, J_{0}=\left[t_{0}, t_{1}\right], J_{k}=\left(t_{k}, t_{k+1}\right]$ for $k=1,2, \ldots, m$. Let $P C(J, R)=\{x$ : $J \rightarrow R ; x(t)$ is continuous everywhere except for some $t_{k}$ at which $x\left(t_{k}^{+}\right)$and $x\left(t_{k}^{-}\right)$exist and $\left.x\left(t_{k}^{-}\right)=x\left(t_{k}\right), k=1,2, \ldots, m\right\}$, and $P C^{1}(J, R)=\left\{x \in P C(J, R) ; x^{\prime}(t)\right.$ is continuous everywhere except for some $t_{k}$ at which $x^{\prime}\left(t_{k}^{+}\right)$and $x^{\prime}\left(t_{k}^{-}\right)$exist and $\left.x^{\prime}\left(t_{k}^{-}\right)=x^{\prime}\left(t_{k}\right), k=1,2, \ldots, m\right\}$. $P C(J$, 
$R)$ and $P C^{1}(J, R)$ are Banach spaces with the norms $\|x\|_{P C}=\sup \{x(t): t \in J\}$ and $\|x\|_{P C^{1}}=$ $\max \left\{\|x\|_{P C},\left\|x^{\prime}\right\|_{P C}\right\}$. Let $E=P C^{1}(J, R) \cap C^{2}\left(J^{-}, R\right)$. A function $x \in E$ is called a solution of PBVP (1.1) if it satisfies (1.1).

Definition 2.1 We say that the functions $\alpha_{0}, \beta_{0} \in E$ are lower and upper solutions of PBVP (1.1), respectively, if there exist $M>0, N \geq 0, L \geq 0, L_{k} \geq 0, L_{k}^{*} \geq 0,0 \leq \varepsilon_{k} \leq \delta_{k} \leq t_{k}-t_{k-1}$, $0 \leq \sigma_{k} \leq \tau_{k} \leq t_{k}-t_{k-1}$, such that

$$
\left\{\begin{array}{l}
\alpha_{0}^{\prime \prime}(t) \geq f\left(t, \alpha_{0}(t),\left(K \alpha_{0}\right)(t),\left(S \alpha_{0}\right)(t)\right)+a(t), \quad t \in J^{-}, \\
\Delta \alpha_{0}\left(t_{k}\right)=I_{k}\left(\int_{t_{k}-\delta_{k}}^{t_{k}-\varepsilon_{k}} \alpha_{0}^{\prime}(s) d s\right)+m_{k}, \quad k=1,2, \ldots, m, \\
\Delta \alpha_{0}^{\prime}\left(t_{k}\right) \geq I_{k}^{*}\left(\int_{t_{k}-\tau_{k}}^{t_{k}-\tau_{k}} \alpha_{0}(s) d s\right)+l_{k}, \quad k=1,2, \ldots, m, \\
\alpha_{0}(0)=\alpha_{0}(T),
\end{array}\right.
$$

where

$$
\begin{aligned}
& a(t)= \begin{cases}0 & \text { if } \alpha_{0}^{\prime}(0) \geq \alpha_{0}^{\prime}(T), \\
\frac{\left[\alpha_{0}^{\prime}(T)-\alpha_{0}^{\prime}(0)\right]}{T}\left[2+M\left[t T-t^{2}\right]+N \int_{0}^{t} k(t, s)\left(s T-s^{2}\right) d s\right. & \text { if } \alpha_{0}^{\prime}(0)<\alpha_{0}^{\prime}(T), \\
\left.+L \int_{0}^{T} h(t, s)\left(s T-s^{2}\right) d s\right] & \text { if } \alpha_{0}^{\prime}(0) \geq \alpha_{0}^{\prime}(T),\end{cases} \\
& m_{k}= \begin{cases}0 & \text { if } \alpha_{0}^{\prime}(0)<\alpha_{0}^{\prime}(T), \\
\frac{L_{k}\left[\alpha_{0}^{\prime}(T)-\alpha_{0}^{\prime}(0)\right]}{T} \int_{t_{k}-\delta_{k}}^{t_{k}-\varepsilon_{k}}(T-2 s) d\end{cases} \\
& l_{k}= \begin{cases}0 & \text { if } \alpha_{0}^{\prime}(0) \geq \alpha_{0}^{\prime}(T), \\
\frac{L_{k}^{*}\left[\alpha_{0}^{\prime}(T)-\alpha_{0}^{\prime}(0)\right]}{T} \int_{t_{k}-\tau_{k}}^{t_{k}-\sigma_{k}}\left(s T-s^{2}\right) d s & \text { if } \alpha_{0}^{\prime}(0)<\alpha_{0}^{\prime}(T),\end{cases}
\end{aligned}
$$

and

$$
\left\{\begin{array}{l}
\beta_{0}^{\prime \prime}(t) \leq f\left(t, \beta_{0}(t),\left(K \beta_{0}\right)(t),\left(S \beta_{0}\right)(t)\right)-b(t), \quad t \in J^{-}, \\
\Delta \beta_{0}\left(t_{k}\right)=I_{k}\left(\int_{t_{k}-\delta_{k}}^{t_{k}-\varepsilon_{k}} \beta_{0}^{\prime}(s) d s\right)-m_{k}^{*}, \quad k=1,2, \ldots, m, \\
\Delta \beta_{0}^{\prime}\left(t_{k}\right) \leq I_{k}^{*}\left(\int_{t_{k}-\tau_{k}}^{t_{k}-\sigma_{k}} \beta_{0}(s) d s\right)-l_{k}^{*}, \quad k=1,2, \ldots, m, \\
\beta_{0}(0)=\beta_{0}(T),
\end{array}\right.
$$

where

$$
\begin{aligned}
& b(t)= \begin{cases}0 & \text { if } \beta_{0}^{\prime}(0) \leq \beta_{0}^{\prime}(T), \\
\frac{\left[\beta_{0}^{\prime}(0)-\beta_{0}^{\prime}(T)\right]}{T}\left[2+M\left[t T-t^{2}\right]+N \int_{0}^{t} k(t, s)\left(s T-s^{2}\right) d s\right. & \text { if } \beta_{0}^{\prime}(0)>\beta_{0}^{\prime}(T), \\
\left.+L \int_{0}^{T} h(t, s)\left(s T-s^{2}\right) d s\right] & \text { if } \beta_{0}^{\prime}(0) \leq \beta_{0}^{\prime}(T),\end{cases} \\
& m_{k}^{*}= \begin{cases}0 & \text { if } \beta_{0}^{\prime}(0)>\beta_{0}^{\prime}(T), \\
\frac{L_{k}\left[\beta_{0}^{\prime}(0)-\beta_{0}^{\prime}(T)\right]}{T} \int_{t_{k}-\delta_{k}}^{t_{k}-\varepsilon_{k}}(T-2 s) \leq \beta_{0}^{\prime}(T),\end{cases} \\
& l_{k}^{*}= \begin{cases}0 & \text { if } \beta_{0}^{\prime}(0)>\beta_{0}^{\prime}(T) . \\
\frac{L_{k}^{*}\left[\beta_{0}^{\prime}(0)-\beta_{0}^{\prime}(T)\right]}{T} \int_{t_{k}-\tau_{k}}^{t_{k}-\sigma_{k}}\left(s T-s^{2}\right) d s\end{cases}
\end{aligned}
$$

Now we are in the position to establish some new comparison principles which play an important role in the monotone iterative technique. 
Lemma 2.1 Assume that $x \in E$ satisfies

$$
\left\{\begin{array}{l}
x^{\prime \prime}(t) \geq M x(t)+N \int_{0}^{t} k(t, s) x(s) d s+L \int_{0}^{T} h(t, s) x(s) d s, \quad t \in J^{-}, \\
\Delta x\left(t_{k}\right)=L_{k} \int_{t_{k}-\delta_{k}}^{t_{k}-\varepsilon_{k}} x^{\prime}(s) d s, \quad k=1,2, \ldots, m, \\
\Delta x^{\prime}\left(t_{k}\right) \geq L_{k}^{*} \int_{t_{k}-\tau_{k}}^{t_{k}-\sigma_{k}} x(s) d s, \quad k=1,2, \ldots, m, \\
x(0)=x(T), \quad x^{\prime}(0) \geq x^{\prime}(T),
\end{array}\right.
$$

where $M>0, N \geq 0, L \geq 0, L_{k} \geq 0, L_{k}^{*} \geq 0$ are constants and $0 \leq \varepsilon_{k} \leq \delta_{k} \leq t_{k}-t_{k-1}, 0 \leq$ $\sigma_{k} \leq \tau_{k} \leq t_{k}-t_{k-1}, k=1,2, \ldots, m$, and they satisfy

$$
\left[\sum_{k=1}^{m} L_{k}\left(\delta_{k}-\varepsilon_{k}\right)+T\right]\left[\sum_{k=1}^{m} L_{k}^{\prime \prime}\left(\tau_{k}-\sigma_{k}\right)+\left(M+N k_{0} T+L h_{0} T\right) T\right] \leq 1 .
$$

Then $x(t) \leq 0, t \in J$.

Proof Suppose, to the contrary, that $x(t)>0$ for some $t \in J$. We divide the proof into two cases:

Case (i). There exists a $\tilde{t} \in J$ such that $x(\tilde{t})>0$ and $x(t) \geq 0$ for all $t \in J$.

From (2.1), we have $x^{\prime \prime}(t) \geq 0$ for $t \in J^{-}$. Since $\Delta x^{\prime}\left(t_{k}\right) \geq L_{k}^{*} \int_{t_{k}-\tau_{k}}^{t_{k}-\tau_{k}} x(s) d s \geq 0$, then $x^{\prime}(t)$ is nondecreasing in $t \in J$ and so $x^{\prime}(0) \leq x^{\prime}(T)$. However, by (2.1) $x^{\prime}(0) \geq x^{\prime}(T)$, then $x^{\prime}(0)=x^{\prime}(T)$, which implies $x^{\prime}(t)=$ constant for all $t \in J$. Thus, $0=x^{\prime \prime}(t) \geq M x(\tilde{t})>0$, a contradiction.

Case (ii). There exists $t^{*}, t_{*} \in J$ such that $x\left(t^{*}\right)>0, x\left(t_{*}\right)<0$.

Let $\inf _{t \in J} x(t)=-\lambda<0$, then there exists $t_{*} \in J_{i}$, for some $i \in\{0,1, \ldots, m\}$, such that $x\left(t_{*}\right)=$ $-\lambda$ or $x\left(t_{i}^{+}\right)=-\lambda$. Without loss of generality, we only consider $x\left(t_{*}\right)=-\lambda$. For the case $x\left(t_{i}^{+}\right)=$ $-\lambda$ the proof is similar. It follows that

$$
\begin{aligned}
x^{\prime \prime}(t) & \geq M x(t)+N \int_{0}^{t} k(t, s) x(s) d s+L \int_{0}^{T} h(t, s) x(s) d s \\
& \geq-\lambda\left(M+N k_{0} T+L h_{0} T\right), \quad t \in J^{-}, \\
\Delta x^{\prime}\left(t_{k}\right) & \geq L_{k}^{\prime \prime} \int_{t_{k}-\tau_{k}}^{t_{k}-\sigma_{k}} x(s) d s \geq-\lambda L_{k}^{\prime \prime}\left(\tau_{k}-\sigma_{k}\right), \quad k=1,2, \ldots, m .
\end{aligned}
$$

If $x^{\prime}(t)>0$ for all $t \in J$, then $\Delta x\left(t_{k}\right)=L_{k} \int_{t_{k}-\delta_{k}}^{t_{k}-\varepsilon_{k}} x^{\prime}(s) d s \geq 0, k=1,2, \ldots, m$. Hence, $x(t)$ is strictly increasing on $J$, which contradicts $x(0)=x(T)$. Then there exists a $\bar{t} \in J$ such that $x^{\prime}(\bar{t}) \leq 0$.

Let $\bar{t} \in J_{j}, j \in\{0,1, \ldots, m\}$. By mean value theorem, we have

$$
\begin{aligned}
x^{\prime}\left(t_{j}^{-}\right)-\lambda L_{j}^{*}\left(\tau_{j}-\sigma_{j}\right)-x^{\prime}(\bar{t}) & \leq x^{\prime}\left(t_{j}^{+}\right)-x^{\prime}(\bar{t})=-x^{\prime \prime}\left(s_{j}\right)\left(\bar{t}-t_{j}^{+}\right) \\
& \leq \lambda\left(M+N k_{0} T+L h_{0} T\right)\left(\bar{t}-t_{j}^{+}\right), \quad s_{j} \in\left(t_{j}, \bar{t}\right), \\
x^{\prime}\left(t_{j-1}^{-}\right)-\lambda L_{j-1}^{*}\left(\tau_{j-1}-\sigma_{j-1}\right)-x^{\prime}\left(t_{j}\right) & \leq x^{\prime}\left(t_{j-1}^{+}\right)-x^{\prime}\left(t_{j}\right)=-x^{\prime \prime}\left(s_{j-1}\right)\left(t_{j}-t_{j-1}^{+}\right) \\
& \leq \lambda\left(M+N k_{0} T+L h_{0} T\right)\left(t_{j}-t_{j-1}^{+}\right), \quad s_{j-1} \in\left(t_{j-1}, t_{j}\right),
\end{aligned}
$$




$$
\begin{aligned}
x^{\prime}\left(t_{1}^{-}\right)-\lambda L_{1}^{*}\left(\tau_{1}-\sigma_{1}\right)-x^{\prime}\left(t_{2}\right) & \leq x^{\prime}\left(t_{1}^{+}\right)-x^{\prime}\left(t_{2}\right)=-x^{\prime \prime}\left(s_{1}\right)\left(t_{2}-t_{1}^{+}\right) \\
& \leq \lambda\left(M+N k_{0} T+L h_{0} T\right)\left(t_{2}-t_{1}^{+}\right), \quad s_{1} \in\left(t_{1}, t_{2}\right), \\
x^{\prime}(0)-x^{\prime}\left(t_{1}\right)=-x^{\prime \prime}\left(s_{0}\right)\left(t_{1}-t_{0}\right) & \leq \lambda\left(M+N k_{0} T+L h_{0} T\right)\left(t_{1}-t_{0}\right), \quad s_{0} \in\left(t_{0}, t_{1}\right) .
\end{aligned}
$$

Summing up the above inequalities, we obtain

$$
\begin{aligned}
x^{\prime}(0) & \leq x^{\prime}(\bar{t})+\lambda\left[\sum_{k=1}^{j} L_{k}^{*}\left(\tau_{k}-\sigma_{k}\right)+\left(M+N k_{0} T+L h_{0} T\right)\left(\bar{t}-t_{0}\right)\right] \\
& \leq \lambda\left[\sum_{k=1}^{j} L_{k}^{*}\left(\tau_{k}-\sigma_{k}\right)+\left(M+N k_{0} T+L h_{0} T\right) \bar{t}\right] .
\end{aligned}
$$

Let $t \in J_{h}, h \in\{0,1, \ldots, m\}$. If $t \leq \bar{t}$ by using the method to get (2.3), then we have

$$
\begin{aligned}
x^{\prime}(t) & \leq x^{\prime}(\bar{t})+\lambda\left[\sum_{k=h+1}^{j} L_{k}^{*}\left(\tau_{k}-\sigma_{k}\right)+\left(M+N k_{0} T+L h_{0} T\right)(\bar{t}-t)\right] \\
& \leq \lambda\left[\sum_{k=1}^{m} L_{k}^{*}\left(\tau_{k}-\sigma_{k}\right)+\left(M+N k_{0} T+L h_{0} T\right) T\right] .
\end{aligned}
$$

If $t>\bar{t}$, then the above method together with (2.1), (2.3) implies that

$$
\begin{aligned}
x^{\prime}(t) \leq & x^{\prime}(T)+\lambda\left[\sum_{k=h+1}^{m} L_{k}^{*}\left(\tau_{k}-\sigma_{k}\right)+\left(M+N k_{0} T+L h_{0} T\right)(T-t)\right] \\
\leq & x^{\prime}(0)+\lambda\left[\sum_{k=h+1}^{m} L_{k}^{*}\left(\tau_{k}-\sigma_{k}\right)+\left(M+N k_{0} T+L h_{0} T\right)(T-t)\right] \\
\leq & \lambda\left[\sum_{k=1}^{j} L_{k}^{*}\left(\tau_{k}-\sigma_{k}\right)+\left(M+N k_{0} T+L h_{0} T\right) \bar{t}\right] \\
& +\lambda\left[\sum_{k=h+1}^{m} L_{k}^{*}\left(\tau_{k}-\sigma_{k}\right)+\left(M+N k_{0} T+L h_{0} T\right)(T-t)\right] \\
\leq & \lambda\left[\sum_{k=1}^{m} L_{k}^{*}\left(\tau_{k}-\sigma_{k}\right)+\left(M+N k_{0} T+L h_{0} T\right) T\right] .
\end{aligned}
$$

Thus,

$$
x^{\prime}(t) \leq \lambda\left[\sum_{k=1}^{m} L_{k}^{*}\left(\tau_{k}-\sigma_{k}\right)+\left(M+N k_{0} T+L h_{0} T\right) T\right], \quad t \in J^{-} .
$$

Let $t^{*} \in J_{r}$ for some $r \in\{0,1, \ldots, m\}$. We first assume that $t^{*}<t^{*}$, then $i<r$. By the mean value theorem, we have

$$
\begin{aligned}
x\left(t^{*}\right)-x\left(t_{r}\right) & =x\left(t^{*}\right)-x\left(t_{r}^{+}\right)+L_{r} \int_{t_{r}-\delta_{r}}^{t_{r}-\varepsilon_{r}} x^{\prime}(s) d s \\
& =x^{\prime}\left(s_{r}\right)\left(t^{*}-t_{r}^{+}\right)+L_{r} \int_{t_{r}-\delta_{r}}^{t_{r}-\varepsilon_{r}} x^{\prime}(s) d s
\end{aligned}
$$




$$
\begin{aligned}
\leq & \left(\left(t^{*}-t_{r}\right)+L_{r}\left(\delta_{r}-\varepsilon_{r}\right)\right) \\
& \times \lambda\left[\sum_{k=1}^{m} L_{k}^{*}\left(\tau_{k}-\sigma_{k}\right)+\left(M+N k_{0} T+L h_{0} T\right) T\right], s_{r} \in\left(t_{r}, t^{*}\right), \\
x\left(t_{r}\right)-x\left(t_{r-1}\right)= & x\left(t_{r}\right)-x\left(t_{r-1}^{+}\right)+L_{r-1} \int_{t_{r-1}-\delta_{r-1}}^{t_{r-1}-\varepsilon_{r-1}} x^{\prime}(s) d s \\
= & x^{\prime}\left(s_{r-1}\right)\left(t_{r}-t_{r-1}^{+}\right)+L_{r-1} \int_{t_{r-1}-\delta_{r-1}}^{t_{r-1}-\varepsilon_{r-1}} x^{\prime}(s) d s \\
\leq & \left(\left(t_{r}-t_{r-1}\right)+L_{r-1}\left(\delta_{r-1}-\varepsilon_{r-1}\right)\right) \\
& \times \lambda\left[\sum_{k=1}^{m} L_{k}^{*}\left(\tau_{k}-\sigma_{k}\right)+\left(M+N k_{0} T+L h_{0} T\right) T\right], \quad s_{r-1} \in\left(t_{r-1}, t_{r}\right), \\
x\left(t_{i+1}\right)-x\left(t_{*}\right) \leq & \left(\left(t_{i+1}-t_{i}\right)+L_{i+1}\left(\delta_{i+1}-\varepsilon_{i+1}\right)\right) \\
& \times \lambda\left[\sum_{k=1}^{m} L_{k}^{*}\left(\tau_{k}-\sigma_{k}\right)+\left(M+N k_{0} T+L h_{0} T\right) T\right] .
\end{aligned}
$$

Summing up, we get

$$
0<x\left(t^{*}\right) \leq-\lambda+\lambda\left[\sum_{k=1}^{m} L_{k}\left(\delta_{k}-\varepsilon_{k}\right)+T\right]\left[\sum_{k=1}^{m} L_{k}^{*}\left(\tau_{k}-\sigma_{k}\right)+\left(M+N k_{0} T+L h_{0} T\right) T\right] .
$$

Hence,

$$
\left[\sum_{k=1}^{m} L_{k}\left(\delta_{k}-\varepsilon_{k}\right)+T\right]\left[\sum_{k=1}^{m} L_{k}^{*}\left(\tau_{k}-\sigma_{k}\right)+\left(M+N k_{0} T+L h_{0} T\right) T\right]>1
$$

which contradicts (2.2).

For the case $t^{*}<t^{*}$, the proof is similar, and thus we omit it. This completes the proof.

Lemma 2.2 Assume that $x \in$ E satisfies

$$
\begin{aligned}
& x^{\prime \prime}(t) \geq M x(t)+N \int_{0}^{t} k(t, s) x(s) d s+L \int_{0}^{T} h(t, s) x(s) d s+\frac{\left[x^{\prime}(T)-x^{\prime}(0)\right]}{T} \\
& \times\left[2+M\left[t T-t^{2}\right]+N \int_{0}^{t} k(t, s)\left(s T-s^{2}\right) d s\right. \\
&\left.+L \int_{0}^{T} h(t, s)\left(s T-s^{2}\right) d s\right], \quad t \in J^{-}, \\
& \Delta x\left(t_{k}\right)= L_{k} \int_{t_{k}-\delta_{k}}^{t_{k}-\varepsilon_{k}} x^{\prime}(s) d s+\frac{L_{k}\left[x^{\prime}(T)-x^{\prime}(0)\right]}{T} \int_{t_{k}-\delta_{k}}^{t_{k}-\varepsilon_{k}}(T-2 s) d s, \quad k=1,2, \ldots, m, \\
& \Delta x^{\prime}\left(t_{k}\right) \geq L_{k}^{*} \int_{t_{k}-\tau_{k}}^{t_{k}-\sigma_{k}} x(s) d s+\frac{L_{k}^{*}\left[x^{\prime}(T)-x^{\prime}(0)\right]}{T} \int_{t_{k}-\tau_{k}}^{t_{k}-\sigma_{k}}\left(s T-s^{2}\right) d s, \quad k=1,2, \ldots, m, \\
& x(0)=x(T), \quad x^{\prime}(0)<x^{\prime}(T),
\end{aligned}
$$


where $M>0, N \geq 0, L \geq 0, L_{k} \geq 0, L_{k}^{*} \geq 0$ are constants and $0 \leq \varepsilon_{k} \leq \delta_{k} \leq t_{k}-t_{k-1}, 0 \leq$ $\sigma_{k} \leq \tau_{k} \leq t_{k}-t_{k-1}, k=1,2, \ldots, m$, and they satisfy (2.2). Then $x(t) \leq 0$ for all $t \in J$.

Proof Let $u(t)=\left[\frac{t T-t^{2}}{T}\right]\left[x^{\prime}(T)-x^{\prime}(0)\right], t \in J$, and define

$$
w(t)=x(t)+u(t)=x(t)+\left[\frac{t T-t^{2}}{T}\right]\left[x^{\prime}(T)-x^{\prime}(0)\right] .
$$

Note that $u(0)=u(T), u(t) \geq 0$ for $t \in J$. If we prove that $w \leq 0$, then $x(t) \leq x(t)+u(t) \leq 0$ and the proof is complete. Since $u^{\prime}(t)=\left[\frac{T-2 t}{T}\right]\left[x^{\prime}(T)-x^{\prime}(0)\right]$, then we get

$$
\begin{aligned}
& w(0)=x(0)+u(0)=x(T)+u(T)=w(T), \\
& w^{\prime}(0)=x^{\prime}(0)+u^{\prime}(0)=x^{\prime}(0)+x^{\prime}(T)-x^{\prime}(0)=x^{\prime}(T), \\
& w^{\prime}(T)=x^{\prime}(T)+u^{\prime}(T)=x^{\prime}(T)-x^{\prime}(T)+x^{\prime}(0)=x^{\prime}(0) .
\end{aligned}
$$

Hence, $w^{\prime}(0)>w^{\prime}(T)$. Indeed, for $k=1,2, \ldots, m$,

$$
\begin{aligned}
\Delta w\left(t_{k}\right) & =\Delta x\left(t_{k}\right)+\Delta u\left(t_{k}\right) \\
& =L_{k} \int_{t_{k}-\delta_{k}}^{t_{k}-\varepsilon_{k}} x^{\prime}(s) d s+\frac{L_{k}\left[x^{\prime}(T)-x^{\prime}(0)\right]}{T} \int_{t_{k}-\delta_{k}}^{t_{k}-\varepsilon_{k}} T-2 s d s \\
& =L_{k} \int_{t_{k}-\delta_{k}}^{t_{k}-\varepsilon_{k}} x^{\prime}(s)+u^{\prime}(s) d s \\
& =L_{k} \int_{t_{k}-\delta_{k}}^{t_{k}-\varepsilon_{k}} w^{\prime}(s) d s,
\end{aligned}
$$

and

$$
\begin{aligned}
\Delta w^{\prime}\left(t_{k}\right) & =\Delta x^{\prime}\left(t_{k}\right)+\Delta u^{\prime}\left(t_{k}\right) \\
& \geq L_{k}^{*} \int_{t_{k}-\tau_{k}}^{t_{k}-\sigma_{k}} x(s) d s+\frac{L_{k}^{*}\left[x^{\prime}(T)-x^{\prime}(0)\right]}{T} \int_{t_{k}-\tau_{k}}^{t_{k}-\sigma_{k}} s T-s^{2} d s \\
& \geq L_{k}^{*} \int_{t_{k}-\tau_{k}}^{t_{k}-\sigma_{k}} x(s)+u(s) d s \\
& \geq L_{k}^{*} \int_{t_{k}-\tau_{k}}^{t_{k}-\sigma_{k}} w(s) d s .
\end{aligned}
$$

Meanwhile, for $t \neq t_{k}, t \in J$,

$$
\begin{aligned}
& w^{\prime \prime}(t)-M w(t)-N \int_{0}^{t} k(t, s) w(s) d s-L \int_{0}^{T} h(t, s) w(s) d s \\
& =x^{\prime \prime}(t)-M x(t)-\frac{M t}{T}\left[x^{\prime}(T)-x^{\prime}(0)\right]-N \int_{0}^{t} k(t, s) x(s) d s-L \int_{0}^{T} h(t, s) x(s) d s \\
& \quad-\frac{\left[x^{\prime}(T)-x^{\prime}(0)\right]}{T}\left[2+M\left[t T-t^{2}\right]+N \int_{0}^{t} k(t, s)\left(s T-s^{2}\right) d s\right. \\
& \left.+L \int_{0}^{T} h(t, s)\left(s T-s^{2}\right) d s\right] \geq 0 .
\end{aligned}
$$

Then by Lemma 2.1, we get $w(t) \leq 0$ for all $t \in J$, which implies that $x(t) \leq 0, t \in J$. 
Consider the linear PBVP

$$
\left\{\begin{array}{l}
x^{\prime \prime}(t)=M x(t)+N \int_{0}^{t} k(t, s) x(s) d s+L \int_{0}^{T} h(t, s) x(s) d s-g(t), \quad t \in J^{-}, \\
\Delta x\left(t_{k}\right)=L_{k} \int_{t_{k}-\delta_{k}}^{t_{k}-\varepsilon_{k}} x^{\prime}(s) d s+\gamma_{k}, \quad k=1,2, \ldots, m, \\
\Delta x^{\prime}\left(t_{k}\right)=L_{k}^{*} \int_{t_{k}-\tau_{k}}^{t_{k}-\sigma_{k}} x(s) d s+\lambda_{k}, \quad k=1,2, \ldots, m, \\
x(0)=x(T), \quad x^{\prime}(0)=x^{\prime}(T),
\end{array}\right.
$$

where constants $M>0, N \geq 0, L \geq 0, L_{k} \geq 0, L_{k}^{*} \geq 0, \gamma_{k}, \lambda_{k}$ are constants and $g \in P C(J, R)$, $0 \leq \varepsilon_{k} \leq \delta_{k} \leq t_{k}-t_{k-1}, 0 \leq \sigma_{k} \leq \tau_{k} \leq t_{k}-t_{k-1}, k=1,2, \ldots, m$.

Lemma 2.3 $x \in E$ is a solution of (2.4) if and only if $x \in P C^{1}(J, R)$ is a solution of the following impulsive integral equation:

$$
\begin{aligned}
x(t)= & \int_{0}^{T} G_{1}(t, s)\left[g(s)-N \int_{0}^{s} k(s, r) x(r) d r-L \int_{0}^{T} h(s, r) x(r) d r\right] d s \\
& +\sum_{k=1}^{m}\left[-G_{1}\left(t, t_{k}\right)\left(L_{k}^{*} \int_{t_{k}-\tau_{k}}^{t_{k}-\sigma_{k}} x(s) d s+\lambda_{k}\right)\right. \\
& \left.+G_{2}\left(t, t_{k}\right)\left(L_{k} \int_{t_{k}-\delta_{k}}^{t_{k}-\varepsilon_{k}} x^{\prime}(s) d s+\gamma_{k}\right)\right],
\end{aligned}
$$

where

$$
\begin{gathered}
G_{1}(t, s)=\left[2 \sqrt{M}\left(e^{\sqrt{M} T}-1\right)\right]^{-1} \begin{cases}e^{\sqrt{M}(T-t+s)}+e^{\sqrt{M}(t-s)}, & 0 \leq s<t \leq T, \\
e^{\sqrt{M}(T+t-s)}+e^{\sqrt{M}(s-t)}, & 0 \leq t \leq s \leq T,\end{cases} \\
G_{2}(t, s)=\left[2\left(e^{\sqrt{M} T}-1\right)\right]^{-1} \begin{cases}e^{\sqrt{M}(T-t+s)}-e^{\sqrt{M}(t-s)}, & 0 \leq s<t \leq T, \\
-e^{\sqrt{M}(T+t-s)}+e^{\sqrt{M}(s-t)}, & 0 \leq t \leq s \leq T .\end{cases}
\end{gathered}
$$

This proof is similar to the proof of Lemma 2.1 in [36], and we omit it.

Lemma 2.4 Let $M>0, N \geq 0, L \geq 0, L_{k} \geq 0, L_{k}^{*} \geq 0$ are constants and $0 \leq \varepsilon_{k} \leq \delta_{k} \leq$ $t_{k}-t_{k-1}, 0 \leq \sigma_{k} \leq \tau_{k} \leq t_{k}-t_{k-1}, k=1,2, \ldots$, m. If

$$
\begin{aligned}
\psi=: & \frac{1+e^{\sqrt{M} T}}{2 \sqrt{M}\left(e^{\sqrt{M} T}-1\right)}\left[\int_{0}^{T}\left[N \int_{0}^{s} k(s, r) d r+L \int_{0}^{T} h(s, r) d r\right] d s+\sum_{k=1}^{m} L_{k}^{*}\left(\tau_{k}-\sigma_{k}\right)\right] \\
& +\frac{1}{2} \sum_{k=1}^{m} L_{k}\left(\delta_{k}-\varepsilon_{k}\right)<1, \\
\mu=: & \frac{1}{2}\left[\int_{0}^{T}\left[N \int_{0}^{s} k(s, r) d r+L \int_{0}^{T} h(s, r) d r\right] d s+\sum_{k=1}^{m} L_{k}^{*}\left(\tau_{k}-\sigma_{k}\right)\right] \\
& +\frac{\sqrt{M}\left(1+e^{\sqrt{M} T}\right)}{2\left(e^{\sqrt{M} T}-1\right)} \sum_{k=1}^{m} L_{k}\left(\delta_{k}-\varepsilon_{k}\right)<1,
\end{aligned}
$$

then (2.4) has a unique solution $x$ in $E$. 
Thaiprayoon et al. Boundary Value Problems 2012, 2012:122

Page 9 of 21

http://www.boundaryvalueproblems.com/content/2012/1/122

Proof For any $x \in E$, we define an operator $F$ by

$$
\begin{aligned}
(F x)(t)= & \int_{0}^{T} G_{1}(t, s)\left[g(s)-N \int_{0}^{s} k(s, r) x(r) d r-L \int_{0}^{T} h(s, r) x(r) d r\right] d s \\
& +\sum_{k=1}^{m}\left[-G_{1}\left(t, t_{k}\right)\left(L_{k}^{*} \int_{t_{k}-\tau_{k}}^{t_{k}-\sigma_{k}} x(s) d s+\lambda_{k}\right)\right. \\
& \left.+G_{2}\left(t, t_{k}\right)\left(L_{k} \int_{t_{k}-\delta_{k}}^{t_{k}-\varepsilon_{k}} x^{\prime}(s) d s+\gamma_{k}\right)\right],
\end{aligned}
$$

where $G_{1}, G_{2}$ are given by Lemma 2.3 . Then $F x \in P C^{1}(J, R)$ and

$$
\begin{aligned}
(F x)^{\prime}(t)= & -\int_{0}^{T} G_{2}(t, s)\left[g(s)-N \int_{0}^{s} k(s, r) x(r) d r-L \int_{0}^{T} h(s, r) x(r) d r\right] d s \\
& +\sum_{k=1}^{m}\left[G_{2}\left(t, t_{k}\right)\left(L_{k}^{*} \int_{t_{k}-\tau_{k}}^{t_{k}-\sigma_{k}} x(s) d s+\lambda_{k}\right)\right. \\
& \left.-M G_{1}\left(t, t_{k}\right)\left(L_{k} \int_{t_{k}-\delta_{k}}^{t_{k}-\varepsilon_{k}} x^{\prime}(s) d s+\gamma_{k}\right)\right] .
\end{aligned}
$$

By computing directly, we have

$$
\max _{(t, s) \in J \times J}\left\{G_{1}(t, s)\right\}=\frac{1+e^{\sqrt{M} T}}{2 \sqrt{M}\left(e^{\sqrt{M} T}-1\right)}
$$

and

$$
\max _{(t, s) \in J \times J}\left\{G_{2}(t, s)\right\}=\frac{1}{2} .
$$

On the other hand, for $x, y \in P C^{1}(J, R)$, we have

$$
\begin{aligned}
\|(F x)-(F y)\|_{P C}= & \sup _{t \in J}|(F x)(t)-(F y)(t)| \\
= & \sup _{t \in J} \mid \int_{0}^{T} G_{1}(t, s)\left[-N \int_{0}^{s} k(s, r)[x(r)-y(r)] d r\right. \\
& \left.-L \int_{0}^{T} h(s, r)[x(r)-y(r)] d r\right] d s \\
& +\sum_{k=1}^{m}\left[-G_{1}\left(t, t_{k}\right)\left(L_{k}^{*} \int_{t_{k}-\tau_{k}}^{t_{k}-\sigma_{k}}[x(s)-y(s)] d s\right)\right. \\
& \left.+G_{2}\left(t, t_{k}\right)\left(L_{k} \int_{t_{k}-\delta_{k}}^{t_{k}-\varepsilon_{k}}\left[x^{\prime}(s)-y^{\prime}(s)\right] d s\right)\right] \mid \\
\leq & \sup _{t \in J} \int_{0}^{T} G_{1}(t, s)\left[N|x(s)-y(s)| \int_{0}^{s} k(s, r) d r\right. \\
& \left.+L|x(s)-y(s)| \int_{0}^{T} h(s, r) d r\right] d s
\end{aligned}
$$




$$
\begin{aligned}
& +\sum_{k=1}^{m}\left[G_{1}\left(t, t_{k}\right) L_{k}^{*}\left(\tau_{k}-\sigma_{k}\right)|x(t)-y(t)|\right. \\
& \left.+G_{2}\left(t, t_{k}\right) L_{k}\left(\delta_{k}-\varepsilon_{k}\right)\left|x^{\prime}(t)-y^{\prime}(t)\right|\right] \\
\leq & \|x-y\|_{P C^{1}}\left[\operatorname { s u p } _ { t \in J } \int _ { 0 } ^ { T } G _ { 1 } ( t , s ) \left[N \int_{0}^{s} k(s, r) d r\right.\right. \\
& \left.+L \int_{0}^{T} h(s, r) d r\right] d s+\sum_{k=1}^{m}\left[G_{1}\left(t, t_{k}\right) L_{k}^{*}\left(\tau_{k}-\sigma_{k}\right)\right. \\
& \left.\left.+G_{2}\left(t, t_{k}\right) L_{k}\left(\delta_{k}-\varepsilon_{k}\right)\right]\right] \\
\leq & \psi\|x-y\|_{P C^{1}} .
\end{aligned}
$$

Similarly,

$$
\begin{aligned}
\left\|(F x)^{\prime}-(F y)^{\prime}\right\|_{P C}= & \sup _{t \in J} \mid-\int_{0}^{T} G_{2}(t, s)\left[-N \int_{0}^{s} k(s, r)[x(r)-y(r)] d r\right. \\
& \left.-L \int_{0}^{T} h(s, r)[x(r)-y(r)] d r\right] d s \\
& +\sum_{k=1}^{m}\left[G_{2}\left(t, t_{k}\right)\left(L_{k}^{*} \int_{t_{k}-\tau_{k}}^{t_{k}-\sigma_{k}}[x(s)-y(s)] d s\right)\right. \\
& \left.-M G_{1}\left(t, t_{k}\right)\left(L_{k} \int_{t_{k}-\delta_{k}}^{t_{k}-\varepsilon_{k}}\left[x^{\prime}(s)-y^{\prime}(s)\right] d s\right)\right] \mid \\
\leq & \sup _{t \in J} \int_{0}^{T} G_{2}(t, s)\left[N|x(s)-y(s)| \int_{0}^{s} k(s, r) d r\right. \\
& \left.+L|x(s)-y(s)| \int_{0}^{T} h(s, r) d r\right] d s \\
& +\sum_{k=1}^{m}\left[G_{2}\left(t, t_{k}\right) L_{k}^{*}\left(\tau_{k}-\sigma_{k}\right)|x(t)-y(t)|\right. \\
& \left.+M G_{1}\left(t, t_{k}\right) L_{k}\left(\delta_{k}-\varepsilon_{k}\right)\left|x^{\prime}(s)-y^{\prime}(s)\right|\right] \\
\leq & \|x-y\|_{P C^{1}}\left[\operatorname { s u p } \int _ { t \in J } ^ { T } G _ { 2 } ( t , s ) \left[N \int_{0}^{s} k(s, r) d r\right.\right. \\
\leq & \mu\|x-y\|_{P C^{1}} . \\
& \left.+L \int_{0}^{T} h(s, r) d r\right] d s+\sum_{k=1}^{m}\left[G_{2}\left(t, t_{k}\right) L_{k}^{*}\left(\tau_{k}-\sigma_{k}\right)\right. \\
& \left.\left.+M G_{1}\left(t, t_{k}\right) L_{k}\left(\delta_{k}-\varepsilon_{k}\right)\right]\right] \\
&
\end{aligned}
$$

Thus,

$$
\|(F x)-(F y)\|_{P C^{1}} \leq \max \{\psi, \mu\}\|x-y\|_{P C^{1}} .
$$


By the Banach fixed-point theorem, $F$ has a unique fixed point $x^{*} \in P C^{1}(J, R)$, and by Lemma $2.3, x^{*}$ is also the unique solution of (2.4). This completes the proof.

\section{Main results}

In this section, we establish existence criteria for solutions of PBVP (1.1) by the method of lower and upper solutions and the monotone iterative technique. For $\alpha_{0}, \beta_{0} \in E$, we write $\alpha_{0} \leq \beta_{0}$ if $\alpha_{0}(t) \leq \beta_{0}(t)$ for all $t \in J$. In such a case, we denote $\left[\alpha_{0}, \beta_{0}\right]=\left\{x \in E: \alpha_{0}(t) \leq\right.$ $\left.x(t) \leq \beta_{0}(t), t \in J\right\}$.

\section{Theorem 3.1 Suppose that the following conditions hold:}

$\left(\mathrm{H}_{1}\right) \quad \alpha_{0}$ and $\beta_{0}$ are lower and upper solutions for $P B V P(1.1)$, respectively, such that $\alpha_{0} \leq \beta_{0}$.

$\left(\mathrm{H}_{2}\right)$ The function $f$ satisfies

$$
f\left(t, x_{2}, y_{2}, z_{2}\right)-f\left(t, x_{1}, y_{1}, z_{1}\right) \leq M\left(x_{2}-x_{1}\right)+N\left(y_{2}-y_{1}\right)+L\left(z_{2}-z_{1}\right)
$$

for all $t \in J, \alpha_{0}(t) \leq x_{1} \leq x_{2} \leq \beta_{0}(t),\left(K \alpha_{0}\right)(t) \leq y_{1} \leq y_{2} \leq\left(K \beta_{0}\right)(t),\left(S \alpha_{0}\right)(t) \leq z_{1} \leq$ $z_{2} \leq\left(S \beta_{0}\right)(t)$.

$\left(\mathrm{H}_{3}\right) M>0, N \geq 0, L \geq 0, L_{k} \geq 0, L_{k}^{*} \geq 0$ are constants, and $0 \leq \varepsilon_{k} \leq \delta_{k} \leq t_{k}-t_{k-1}, 0 \leq$ $\sigma_{k} \leq \tau_{k} \leq t_{k}-t_{k-1}, k=1,2, \ldots, m$, and they satisfy (2.2), (2.6) and (2.7).

$\left(\mathrm{H}_{4}\right)$ The functions $I_{k}, I_{k}^{*}$ satisfy

$$
\begin{aligned}
& I_{k}\left(\int_{t_{k}-\delta_{k}}^{t_{k}-\varepsilon_{k}} x^{\prime}(s) d s\right)-I_{k}\left(\int_{t_{k}-\delta_{k}}^{t_{k}-\varepsilon_{k}} y^{\prime}(s) d s\right)=L_{k} \int_{t_{k}-\delta_{k}}^{t_{k}-\varepsilon_{k}} x^{\prime}(s)-y^{\prime}(s) d s, \\
& I_{k}^{*}\left(\int_{t_{k}-\tau_{k}}^{t_{k}-\sigma_{k}} x(s) d s\right)-I_{k}^{*}\left(\int_{t_{k}-\tau_{k}}^{t_{k}-\sigma_{k}} y(s) d s\right) \leq L_{k}^{*} \int_{t_{k}-\tau_{k}}^{t_{k}-\sigma_{k}} x(s)-y(s) d s,
\end{aligned}
$$

where $\int_{t_{k}-\tau_{k}}^{t_{k}-\sigma_{k}} \alpha_{0}(s) d s \leq \int_{t_{k}-\tau_{k}}^{t_{k}-\sigma_{k}} y(s) d s \leq \int_{t_{k}-\tau_{k}}^{t_{k}-\sigma_{k}} x(s) d s \leq \int_{t_{k}-\tau_{k}}^{t_{k}-\sigma_{k}} \beta_{0}(s) d s, 0 \leq \sigma_{k} \leq \tau_{k} \leq$ $t_{k}-t_{k-1}, 0 \leq \varepsilon_{k} \leq \delta_{k} \leq t_{k}-t_{k-1}, k=1,2, \ldots, m$.

Then there exist monotone sequences $\left\{\alpha_{n}\right\},\left\{\beta_{n}\right\} \subset E$ which converge in $E$ to the extreme solutions of PBVP (1.1) in $\left[\alpha_{0}, \beta_{0}\right]$, respectively.

Proof For any $\eta \in\left[\alpha_{0}, \beta_{0}\right]$, we consider linear PBVP (2.4) with

$$
\begin{aligned}
& g(t)=f(t, \eta(t),(K \eta)(t),(S \eta)(t))-M \eta(t)-N \int_{0}^{t} k(t, s) \eta(s) d s-L \int_{0}^{T} h(t, s) \eta(s) d s, \\
& \gamma_{k}=I_{k}\left(\int_{t_{k}-\delta_{k}}^{t_{k}-\varepsilon_{k}} \eta^{\prime}(s) d s\right)-L_{k} \int_{t_{k}-\delta_{k}}^{t_{k}-\varepsilon_{k}} \eta^{\prime}(s) d s, \quad k=1,2, \ldots, m, \\
& \lambda_{k}=I_{k}^{*}\left(\int_{t_{k}-\tau_{k}}^{t_{k}-\sigma_{k}} \eta(s) d s\right)-L_{k}^{*} \int_{t_{k}-\tau_{k}}^{t_{k}-\sigma_{k}} \eta(s) d s, \quad k=1,2, \ldots, m .
\end{aligned}
$$

By Lemma 2.4, PBVP (2.4) has a unique solution $x \in E$. We define an operator $A$ from $\left[\alpha_{0}, \beta_{0}\right]$ to $E$ by $x(t)=A \eta(t)$. We complete the proof in four steps.

Step 1 . We claim that $\alpha_{0} \leq A \alpha_{0}$ and $A \beta_{0} \leq \beta_{0}$. We only prove $\alpha_{0} \leq A \alpha_{0}$ since the second inequality can be proved in a similar manner. 
Let $\alpha_{1}=A \alpha_{0}$ and $p=\alpha_{0}-\alpha_{1}$. Then $\alpha_{1}$ satisfies

$$
\begin{gathered}
\alpha_{1}^{\prime \prime}(t)-M \alpha_{1}(t)-N\left(K \alpha_{1}\right)(t)-L\left(S \alpha_{1}\right)(t) \\
=f\left(t, \alpha_{0}(t),\left(K \alpha_{0}\right)(t),\left(S \alpha_{0}\right)(t)\right)-M \alpha_{0}(t)-N\left(K \alpha_{0}\right)(t) \\
-L\left(S \alpha_{0}\right)(t), \quad t \in J^{-}, \\
\Delta \alpha_{1}\left(t_{k}\right)=L_{k} \int_{t_{k}-\delta_{k}}^{t_{k}-\varepsilon_{k}} \alpha_{1}^{\prime}(s) d s+I_{k}\left(\int_{t_{k}-\delta_{k}}^{t_{k}-\varepsilon_{k}} \alpha_{0}^{\prime}(s) d s\right) \\
\quad-L_{k} \int_{t_{k}-\delta_{k}}^{t_{k}-\varepsilon_{k}} \alpha_{0}^{\prime}(s) d s, \quad k=1,2, \ldots, m, \\
\Delta \alpha_{1}^{\prime}\left(t_{k}\right)=L_{k}^{*} \int_{t_{k}-\tau_{k}}^{t_{k}-\sigma_{k}} \alpha_{1}(s) d s+I_{k}^{*}\left(\int_{t_{k}-\tau_{k}}^{t_{k}-\sigma_{k}} \alpha_{0}(s) d s\right) \\
-L_{k}^{*} \int_{t_{k}-\tau_{k}}^{t_{k}-\sigma_{k}} \alpha_{0}(s) d s, \quad k=1,2, \ldots, m, \\
\alpha_{1}(0)=\alpha_{1}(T), \quad \alpha_{1}^{\prime}(0)=\alpha_{1}^{\prime}(T) .
\end{gathered}
$$

We finish Step 1 in two cases.

Case 1. $\alpha_{0}^{\prime}(0) \geq \alpha_{0}^{\prime}(T)$, which implies that

$$
a(t)=0, \quad \alpha_{0}^{\prime \prime}(t) \geq f\left(t, \alpha_{0}(t),\left(K \alpha_{0}\right)(t),\left(S \alpha_{0}\right)(t)\right) .
$$

As $\alpha_{0}$ is a lower solution of PBVP (1.1), then for $t \in J^{-}$,

$$
\begin{aligned}
p^{\prime \prime}(t) & -M p(t)-N(K p)(t)-L(S p)(t) \\
= & \alpha_{0}^{\prime \prime}(t)-M \alpha_{0}(t)-N\left(K \alpha_{0}\right)(t)-L\left(S \alpha_{0}\right)(t) \\
& \quad-\alpha_{1}^{\prime \prime}(t)+M \alpha_{1}(t)+N\left(K \alpha_{1}\right)(t)+L\left(S \alpha_{1}\right)(t) \\
\geq & f\left(t, \alpha_{0}(t),\left(K \alpha_{0}\right)(t),\left(S \alpha_{0}\right)(t)\right)-M \alpha_{0}(t)-N\left(K \alpha_{0}\right)(t) \\
& \quad-L\left(S \alpha_{0}\right)(t)-f\left(t, \alpha_{0}(t),\left(K \alpha_{0}\right)(t),\left(S \alpha_{0}\right)(t)\right)+M \alpha_{0}(t) \\
& +N\left(K \alpha_{0}\right)(t)+L\left(S \alpha_{0}\right)(t) \\
= & 0
\end{aligned}
$$

and

$$
\begin{aligned}
\Delta p\left(t_{k}\right)= & \Delta \alpha_{0}\left(t_{k}\right)-\alpha_{1}\left(t_{k}\right) \\
= & I_{k}\left(\int_{t_{k}-\delta_{k}}^{t_{k}-\varepsilon_{k}} \alpha_{0}^{\prime}(s) d s\right)-L_{k} \int_{t_{k}-\delta_{k}}^{t_{k}-\varepsilon_{k}} \alpha_{1}^{\prime}(s) d s-I_{k}\left(\int_{t_{k}-\delta_{k}}^{t_{k}-\varepsilon_{k}} \alpha_{0}^{\prime}(s) d s\right) \\
& +L_{k} \int_{t_{k}-\delta_{k}}^{t_{k}-\varepsilon_{k}} \alpha_{0}^{\prime}(s) d s \\
= & L_{k} \int_{t_{k}-\delta_{k}}^{t_{k}-\varepsilon_{k}} \alpha_{0}^{\prime}(s)-\alpha_{1}^{\prime}(s) d s=L_{k} \int_{t_{k}-\delta_{k}}^{t_{k}-\varepsilon_{k}} p^{\prime}(s) d s, \quad k=1,2, \ldots, m, \\
\Delta p^{\prime}\left(t_{k}\right)= & \Delta \alpha_{0}^{\prime}\left(t_{k}\right)-\alpha_{1}^{\prime}\left(t_{k}\right)
\end{aligned}
$$




$$
\begin{aligned}
& \geq I_{k}^{\prime \prime}\left(\int_{t_{k}-\tau_{k}}^{t_{k}-\sigma_{k}} \alpha_{0}(s) d s\right)-L_{k}^{*} \int_{t_{k}-\tau_{k}}^{t_{k}-\sigma_{k}} \alpha_{1}(s) d s-I_{k}^{*}\left(\int_{t_{k}-\tau_{k}}^{t_{k}-\sigma_{k}} \alpha_{0}(s) d s\right) \\
&+L_{k}^{*} \int_{t_{k}-\tau_{k}}^{t_{k}-\sigma_{k}} \alpha_{0}(s) d s \\
&=L_{k}^{*} \int_{t_{k}-\tau_{k}}^{t_{k}-\sigma_{k}} \alpha_{0}(s)-\alpha_{1}(s) d s=L_{k}^{*} \int_{t_{k}-\tau_{k}}^{t_{k}-\sigma_{k}} p(s) d s, \quad k=1,2, \ldots, m, \\
& p(0)=\alpha_{0}(0)-\alpha_{1}(0)=\alpha_{0}(T)-\alpha_{1}(T)=p(T), \\
& p^{\prime}(0)=\alpha_{0}^{\prime}(0)-\alpha_{1}^{\prime}(0) \geq \alpha_{0}^{\prime}(T)-\alpha_{1}^{\prime}(T)=p^{\prime}(T) .
\end{aligned}
$$

Then by Lemma 2.1, $p(t) \leq 0$, which implies that $\alpha_{0}(t) \leq A \alpha_{0}(t)$, i.e., $\alpha_{0} \leq A \alpha_{0}$.

Case 2. $\alpha_{0}^{\prime}(0)<\alpha_{0}^{\prime}(T)$, which implies that

$$
\begin{aligned}
a(t)= & \frac{\left[\alpha_{0}^{\prime}(T)-\alpha_{0}^{\prime}(0)\right]}{T}\left[2+M\left[t T-t^{2}\right]+N \int_{0}^{t} k(t, s)\left(s T-s^{2}\right) d s\right. \\
& \left.+L \int_{0}^{T} h(t, s)\left(s T-s^{2}\right) d s\right] .
\end{aligned}
$$

Hence,

$$
\begin{aligned}
p^{\prime \prime}(t) & -M p(t)-N(K p)(t)-L(S p)(t)-\frac{\left[p^{\prime}(T)-p^{\prime}(0)\right]}{T}\left[2+M\left[t T-t^{2}\right]\right. \\
& \left.+N \int_{0}^{t} k(t, s)\left(s T-s^{2}\right) d s+L \int_{0}^{T} h(t, s)\left(s T-s^{2}\right) d s\right] \\
= & \alpha_{0}^{\prime \prime}(t)-M \alpha_{0}(t)-N\left(K \alpha_{0}\right)(t)-L\left(S \alpha_{0}\right)(t)-\frac{\left[\alpha_{0}^{\prime}(T)-\alpha_{0}^{\prime}(0)\right]}{T}\left[2+M\left[t T-t^{2}\right]\right. \\
& \left.+N \int_{0}^{t} k(t, s)\left(s T-s^{2}\right) d s+L \int_{0}^{T} h(t, s)\left(s T-s^{2}\right) d s\right] \\
& -\alpha_{1}^{\prime \prime}(t)+M \alpha_{1}(t)+N\left(K \alpha_{1}\right)(t)+L\left(S \alpha_{1}\right)(t) \\
\geq & f\left(t, \alpha_{0}(t),\left(K \alpha_{0}\right)(t),\left(S \alpha_{0}\right)(t)\right)-M \alpha_{0}(t)-N\left(K \alpha_{0}\right)(t) \\
& -L\left(S \alpha_{0}\right)(t)-f\left(t, \alpha_{0}(t),\left(K \alpha_{0}\right)(t),\left(S \alpha_{0}\right)(t)\right) \\
& +M \alpha_{0}(t)+N\left(K \alpha_{0}\right)(t)+L\left(S \alpha_{0}\right)(t)=0,
\end{aligned}
$$

and

$$
\begin{aligned}
\Delta p\left(t_{k}\right)= & \Delta \alpha_{0}\left(t_{k}\right)-\Delta \alpha_{1}\left(t_{k}\right) \\
= & I_{k}\left(\int_{t_{k}-\delta_{k}}^{t_{k}-\varepsilon_{k}} \alpha_{0}^{\prime}(s) d s\right)+\frac{L_{k}\left[\alpha_{0}^{\prime}(T)-\alpha_{0}^{\prime}(0)\right]}{T} \int_{t_{k}-\delta_{k}}^{t_{k}-\varepsilon_{k}} T-2 s d s \\
& -L_{k} \int_{t_{k}-\delta_{k}}^{t_{k}-\varepsilon_{k}} \alpha_{1}^{\prime}(s) d s-I_{k}\left(\int_{t_{k}-\delta_{k}}^{t_{k}-\varepsilon_{k}} \alpha_{0}^{\prime}(s) d s\right)+L_{k} \int_{t_{k}-\delta_{k}}^{t_{k}-\varepsilon_{k}} \alpha_{0}^{\prime}(s) d s \\
= & L_{k} \int_{t_{k}-\delta_{k}}^{t_{k}-\varepsilon_{k}} p^{\prime}(s) d s+\frac{L_{k}\left[p^{\prime}(T)-p^{\prime}(0)\right]}{T} \int_{t_{k}-\delta_{k}}^{t_{k}-\varepsilon_{k}} T-2 s d s, \quad k=1,2, \ldots, m,
\end{aligned}
$$


and

$$
\begin{aligned}
\Delta p^{\prime}\left(t_{k}\right)= & \Delta \alpha_{0}^{\prime}\left(t_{k}\right)-\alpha_{1}^{\prime}\left(t_{k}\right) \\
\geq & I_{k}^{*}\left(\int_{t_{k}-\tau_{k}}^{t_{k}-\sigma_{k}} \alpha_{0}(s) d s\right)+\frac{L_{k}^{*}\left[\alpha_{0}^{\prime}(T)-\alpha_{0}^{\prime}(0)\right]}{T} \int_{t_{k}-\tau_{k}}^{t_{k}-\sigma_{k}} s T-s^{2} d s \\
& -L_{k}^{*} \int_{t_{k}-\tau_{k}}^{t_{k}-\sigma_{k}} \alpha_{1}(s) d s-I_{k}^{*}\left(\int_{t_{k}-\tau_{k}}^{t_{k}-\sigma_{k}} \alpha_{0}(s) d s\right)+L_{k}^{*} \int_{t_{k}-\tau_{k}}^{t_{k}-\sigma_{k}} \alpha_{0}(s) d s \\
= & L_{k}^{*} \int_{t_{k}-\tau_{k}}^{t_{k}-\sigma_{k}} p(s) d s+\frac{L_{k}^{*}\left[p^{\prime}(T)-p^{\prime}(0)\right]}{T} \int_{t_{k}-\tau_{k}}^{t_{k}-\sigma_{k}} s T-s^{2} d s,
\end{aligned}
$$

$k=1,2, \ldots, m$, and

$$
p(0)=p(T), \quad p^{\prime}(0)=\alpha_{0}^{\prime}(0)-\alpha_{1}^{\prime}(0)<\alpha_{0}^{\prime}(T)-\alpha_{1}^{\prime}(T)=p^{\prime}(T) .
$$

Then by Lemma 2.2, $p(t) \leq 0$, which implies $\alpha_{0}(t) \leq A \alpha_{0}(t)$, i.e., $\alpha_{0} \leq A \alpha_{0}$.

Step 2. We prove that if $\alpha_{0} \leq \eta_{1} \leq \eta_{2} \leq \beta_{0}$, then $A \eta_{1} \leq A \eta_{2}$.

Let $\eta_{1}^{*}=A \eta_{1}, \eta_{2}^{*}=A \eta_{2}$, and $p=\eta_{1}^{*}-\eta_{2}^{*}$, then for $t \in J^{-}$, and by $\left(\mathrm{H}_{2}\right)$, we obtain

$$
\begin{aligned}
p^{\prime \prime}(t) & -M p(t)-N(K p)(t)-L(S p)(t) \\
= & f\left(t, \eta_{1}(t),\left(K \eta_{1}\right)(t),\left(S \eta_{1}\right)(t)\right)-M \eta_{1}(t)-N\left(K \eta_{1}\right)(t) \\
& \quad-L\left(S \eta_{1}\right)(t)-f\left(t, \eta_{2}(t),\left(K \eta_{2}\right)(t),\left(S \eta_{2}\right)(t)\right) \\
& +M \eta_{2}(t)+N\left(K \eta_{2}\right)(t)+L\left(S \eta_{2}\right)(t) \\
\geq & 0 \quad\left(\text { by }\left(\mathrm{H}_{2}\right)\right) .
\end{aligned}
$$

From $\left(\mathrm{H}_{3}\right)$, we obtain

$$
\begin{aligned}
& \Delta p\left(t_{k}\right)=L_{k} \int_{t_{k}-\delta_{k}}^{t_{k}-\varepsilon_{k}} p^{\prime}(s) d s, \quad k=1,2, \ldots, m, \\
& \Delta p^{\prime}\left(t_{k}\right) \geq L_{k}^{*} \int_{t_{k}-\tau_{k}}^{t_{k}-\sigma_{k}} p(s) d s, \quad k=1,2, \ldots, m, \\
& p(0)=p(T), \quad p^{\prime}(0)=p^{\prime}(T) .
\end{aligned}
$$

Applying Lemma 2.1, we get $p(t) \leq 0$, which implies $A \eta_{1} \leq A \eta_{2}$.

Step 3. We show that PBVP (1.1) has solutions.

Let $\alpha_{n}=A \alpha_{n-1}, \beta_{n}=A \beta_{n-1}, n=1,2, \ldots$. Following the first two steps, we have

$$
\alpha_{0} \leq \alpha_{1} \leq \cdots \leq \alpha_{n} \leq \cdots \leq \beta_{n} \leq \cdots \leq \beta_{1} \leq \beta_{0}, \quad \forall n \in N
$$

Obviously, each $\alpha_{i}, \beta_{i}(i=1,2, \ldots)$ satisfies

$$
\begin{aligned}
\alpha_{i}^{\prime \prime}(t) & -M \alpha_{i}(t)-N\left(K \alpha_{i}\right)(t)-L\left(S \alpha_{i}\right)(t) \\
= & f\left(t, \alpha_{i-1}(t),\left(K \alpha_{i-1}\right)(t),\left(S \alpha_{i-1}\right)(t)\right)-M \alpha_{i-1}(t)-N\left(K \alpha_{i-1}\right)(t) \\
& \quad-L\left(S \alpha_{i-1}\right)(t), \quad t \in J^{-}
\end{aligned}
$$




$$
\begin{aligned}
& \Delta \alpha_{i}\left(t_{k}\right)= L_{k} \int_{t_{k}-\delta_{k}}^{t_{k}-\varepsilon_{k}} \alpha_{i}^{\prime}(s) d s+I_{k}\left(\int_{t_{k}-\delta_{k}}^{t_{k}-\varepsilon_{k}} \alpha_{i-1}^{\prime}(s) d s\right) \\
&-L_{k} \int_{t_{k}-\delta_{k}}^{t_{k}-\varepsilon_{k}} \alpha_{i-1}^{\prime}(s) d s, \quad k=1,2, \ldots, m, \\
& \Delta \alpha_{i}^{\prime}\left(t_{k}\right)= L_{k}^{*} \int_{t_{k}-\tau_{k}}^{t_{k}-\sigma_{k}} \alpha_{i}(s) d s+I_{k}^{*}\left(\int_{t_{k}-\tau_{k}}^{t_{k}-\sigma_{k}} \alpha_{i-1}(s) d s\right) \\
&-L_{k}^{*} \int_{t_{k}-\tau_{k}}^{t_{k}-\sigma_{k}} \alpha_{i-1}(s) d s, \quad k=1,2, \ldots, m, \\
& \alpha_{i}(0)=\alpha_{i}(T), \quad \alpha_{i}^{\prime}(0)=\alpha_{i}^{\prime}(T),
\end{aligned}
$$

and

$$
\begin{aligned}
& \beta_{i}^{\prime \prime}(t)-M \beta_{i}(t)-N\left(K \beta_{i}\right)(t)-L\left(S \beta_{i}\right)(t) \\
& =f\left(t, \beta_{i-1}(t),\left(K \beta_{i-1}\right)(t),\left(S \beta_{i-1}\right)(t)\right)-M \beta_{i-1}(t)-N\left(K \beta_{i-1}\right)(t) \\
& -L\left(S \beta_{i-1}\right)(t), \quad t \in J^{-}, \\
& \Delta \beta_{i}\left(t_{k}\right)=L_{k} \int_{t_{k}-\delta_{k}}^{t_{k}-\varepsilon_{k}} \beta_{i}^{\prime}(s) d s+I_{k}\left(\int_{t_{k}-\delta_{k}}^{t_{k}-\varepsilon_{k}} \beta_{i-1}^{\prime}(s) d s\right) \\
& -L_{k} \int_{t_{k}-\delta_{k}}^{t_{k}-\varepsilon_{k}} \beta_{i-1}^{\prime}(s) d s, \quad k=1,2, \ldots, m, \\
& \Delta \beta_{i}^{\prime}\left(t_{k}\right)=L_{k}^{*} \int_{t_{k}-\tau_{k}}^{t_{k}-\sigma_{k}} \beta_{i}(s) d s+I_{k}^{*}\left(\int_{t_{k}-\tau_{k}}^{t_{k}-\sigma_{k}} \beta_{i-1}(s) d s\right) \\
& -L_{k}^{*} \int_{t_{k}-\tau_{k}}^{t_{k}-\sigma_{k}} \beta_{i-1}(s) d s, \quad k=1,2, \ldots, m, \\
& \beta_{i}(0)=\beta_{i}(T), \quad \beta_{i}^{\prime}(0)=\beta_{i}^{\prime}(T) .
\end{aligned}
$$

Thus, there exist $x$ and $x^{*}$ such that

$$
\lim _{i \rightarrow \infty} \alpha_{i}(t)=x_{*}(t), \quad \lim _{i \rightarrow \infty} \beta_{i}(t)=x^{*}(t), \quad \text { uniformly on } t \in J .
$$

Clearly, $x_{*}^{*}, x^{*}$ satisfy PBVP (1.1).

Step 4 . We show that $x^{*}, x^{*}$ are extreme solutions of PBVP (1.1).

Let $x(t)$ be any solution of PBVP (1.1), which satisfies $\alpha_{0}(t) \leq x(t) \leq \beta_{0}(t), t \in J$. Suppose that there exists a positive integer $n$ such that for $t \in J, \alpha_{n}(t) \leq x(t) \leq \beta_{n}(t)$. Setting $p(t)=$ $\alpha_{n+1}(t)-x(t)$, then for $t \in J^{-}$,

$$
\begin{aligned}
p^{\prime \prime}(t)= & \alpha_{n+1}^{\prime \prime}-x^{\prime \prime}(t) \\
= & M \alpha_{n+1}(t)+N\left(K \alpha_{n+1}\right)(t)+L\left(S \alpha_{n+1}\right)(t) \\
& +f\left(t, \alpha_{n}(t),\left(K \alpha_{n}\right)(t),\left(S \alpha_{n}\right)(t)\right)-M \alpha_{n}(t) \\
& -N\left(K \alpha_{n}\right)(t)-L\left(S \alpha_{n}\right)(t) \\
& -f(t, x(t),(K x)(t),(S x)(t)) \\
= & M \alpha_{n+1}(t)+N\left(K \alpha_{n+1}\right)(t)+L\left(S \alpha_{n+1}\right)(t)-M x(t)
\end{aligned}
$$




$$
\begin{aligned}
& -N(K x)(t)-L(S x)(t)+f\left(t, \alpha_{n}(t),\left(K \alpha_{n}\right)(t),\left(S \alpha_{n}\right)(t)\right) \\
& -f(t, x(t),(K x)(t),(S x)(t))-M\left(\alpha_{n}(t)-x(t)\right) \\
& -N\left(K\left(\alpha_{n}\right)(t)-(K x)(t)\right)-L\left(\left(S \alpha_{n}\right)(t)-(S x)(t)\right) \\
\geq & M p(t)+N(K p)(t)+L(S p)(t),
\end{aligned}
$$

and

$$
\Delta p\left(t_{k}\right)=\Delta \alpha_{n+1}\left(t_{k}\right)-\Delta x\left(t_{k}\right)=L_{k} \int_{t_{k}-\delta_{k}}^{t_{k}-\varepsilon_{k}} p^{\prime}(s) d s, \quad k=1,2, \ldots, m
$$

and

$$
\begin{aligned}
\Delta p^{\prime}\left(t_{k}\right)= & \Delta \alpha_{n+1}^{\prime}\left(t_{k}\right)-\Delta x^{\prime}\left(t_{k}\right) \\
= & L_{k}^{*} \int_{t_{k}-\tau_{k}}^{t_{k}-\sigma_{k}} \alpha_{n+1}(s) d s+I_{k}^{*}\left(\int_{t_{k}-\tau_{k}}^{t_{k}-\sigma_{k}} \alpha_{n}(s) d s\right) \\
& -L_{k}^{*} \int_{t_{k}-\tau_{k}}^{t_{k}-\sigma_{k}} \alpha_{n}(s) d s-I_{k}^{*}\left(\int_{t_{k}-\tau_{k}}^{t_{k}-\sigma_{k}} x(s) d s\right) \\
\geq & L_{k}^{*} \int_{t_{k}-\tau_{k}}^{t_{k}-\sigma_{k}} \alpha_{n+1}(s) d s+L_{k}^{*} \int_{t_{k}-\tau_{k}}^{t_{k}-\sigma_{k}} \alpha_{n}(s) d s \\
& -L_{k}^{*} \int_{t_{k}-\tau_{k}}^{t_{k}-\sigma_{k}} \alpha_{n}(s) d s-L_{k}^{*} \int_{t_{k}-\tau_{k}}^{t_{k}-\sigma_{k}} x(s) d s \\
= & L_{k}^{*} \int_{t_{k}-\tau_{k}}^{t_{k}-\sigma_{k}} p(s) d s, \quad k=1,2, \ldots, m,
\end{aligned}
$$

and

$$
p(0)=p(T), \quad p^{\prime}(0)=p^{\prime}(T) .
$$

Still by Lemma 2.1, we have for all $t \in J, p(t) \leq 0$, i.e., $\alpha_{n+1}(t) \leq x(t)$. Similarly, we can prove that $x(t) \leq \beta_{n+1}(t), t \in J$. Therefore, $\alpha_{n+1}(t) \leq x(t) \leq \beta_{n+1}(t)$, for all $t \in J$, which implies $x=(t) \leq x(t) \leq x^{*}(t)$. The proof is complete.

\section{An example}

In this section, in order to illustrate our results, we consider an example.

Example 4.1 Consider the following PBVP:

$$
\left\{\begin{aligned}
& u^{\prime \prime}(t)= \frac{1}{4} t^{3}(u(t)-2)+\frac{5}{18}\left[\int_{0}^{t} t^{2} s^{4} u(s) d s\right]^{2} \\
&+\frac{1}{8}\left[\int_{0}^{1} t^{3} s^{2} u(s) d s\right]^{2}, \quad t \in J=[0,1], t \neq \frac{1}{2}, \\
& \Delta u\left(\frac{1}{2}\right)= \frac{1}{2} \int_{\frac{1}{6}}^{\frac{3}{10}} u^{\prime}(s) d s, \quad k=1, \\
& \Delta u^{\prime}\left(\frac{1}{2}\right)=\frac{1}{3} \int_{\frac{1}{10}}^{\frac{3}{10}} u(s) d s, \quad k=1, \\
& u(0)=u(1), \quad u^{\prime}(0)=u^{\prime}(1) .
\end{aligned}\right.
$$


Set $k(t, s)=t^{2} s^{4}, h(t, s)=t^{3} s^{2}, m=1, t_{1}=\frac{1}{2}, \delta_{1}=\frac{1}{3}, \varepsilon_{1}=\frac{1}{5}, \tau_{1}=\frac{2}{5}, \sigma_{1}=\frac{1}{5}, T=1$. Obviously, $\alpha_{0}=0, \beta_{0}=3$ are lower and upper solutions for (4.1), respectively, and $\alpha_{0} \leq \beta_{0}$.

Let

$$
f\left(t, x_{1}, y_{1}, z_{1}\right)=\frac{1}{4} t^{3}\left(x_{1}-2\right)+\frac{5}{18} y_{1}^{2}+\frac{1}{8} z_{1}^{2},
$$

we have

$$
f\left(t, x_{2}, y_{2}, z_{2}\right)-f\left(t, x_{1}, y_{1}, z_{1}\right) \leq \frac{1}{4}\left(x_{2}-x_{1}\right)+\frac{1}{3}\left(y_{2}-y_{1}\right)+\frac{1}{4}\left(z_{2}-z_{1}\right),
$$

where $\alpha(t) \leq x_{1} \leq x_{2} \leq \beta(t),(K \alpha)(t) \leq y_{1} \leq y_{2} \leq(K \beta)(t),(S \alpha)(t) \leq z_{1} \leq z_{2} \leq(S \beta)(t), t \in J$. It is easy to see that

$$
I_{1}\left(\int_{\frac{1}{6}}^{\frac{3}{10}} x^{\prime}(s) d s\right)-I_{1}\left(\int_{\frac{1}{6}}^{\frac{3}{10}} y^{\prime}(s) d s\right)=\frac{1}{2} \int_{\frac{1}{6}}^{\frac{3}{10}} x^{\prime}(s)-y^{\prime}(s) d s,
$$

and

$$
I_{1}^{\prime \prime}\left(\int_{\frac{1}{10}}^{\frac{3}{10}} x(s) d s\right)-I_{1}^{\prime \prime}\left(\int_{\frac{1}{10}}^{\frac{3}{10}} y(s) d s\right)=\frac{1}{3} \int_{\frac{1}{10}}^{\frac{3}{10}} x(s)-y(s) d s,
$$

whenever $\int_{t_{k}-\tau_{k}}^{t_{k}-\sigma_{k}} \alpha(s) d s \leq \int_{t_{k}-\tau_{k}}^{t_{k}-\sigma_{k}} y(s) d s \leq \int_{t_{k}-\tau_{k}}^{t_{k}-\sigma_{k}} x(s) d s \leq \int_{t_{k}-\tau_{k}}^{t_{k}-\sigma_{k}} \beta(s) d s, k=1$.

Taking $M=\frac{1}{4}, N=\frac{1}{3}, L=\frac{1}{4}, L_{1}=\frac{1}{2}, L_{1}^{*}=\frac{1}{3}$, it follows that

$$
\begin{aligned}
& {\left[\sum_{k=1}^{m} L_{k}\left(\delta_{k}-\varepsilon_{k}\right)+T\right]\left[\sum_{k=1}^{m} L_{k}^{*}\left(\tau_{k}-\sigma_{k}\right)+\left(M+N k_{0} T+L h_{0} T\right) T\right]} \\
& \quad=\left[\frac{1}{2}\left(\frac{1}{3}-\frac{1}{5}\right)+1\right]\left[\frac{1}{3}\left(\frac{2}{5}-\frac{1}{5}\right)+\left(\frac{1}{4}+\left(\frac{1}{3}\right)(1)(1)+\left(\frac{1}{4}\right)(1)(1)\right) 1\right] \\
& \quad=\frac{24}{25} \leq 1,
\end{aligned}
$$

and

$$
\begin{aligned}
\psi= & \frac{1+e^{\sqrt{M} T}}{2 \sqrt{M}\left(e^{\sqrt{M} T}-1\right)} \\
& \times\left[\int_{0}^{T}\left[N \int_{0}^{s} k(s, r) d r+L \int_{0}^{T} h(s, r) d r\right] d s+\sum_{k=1}^{m} L_{k}^{*}\left(\tau_{k}-\sigma_{k}\right)\right] \\
& +\frac{1}{2} \sum_{k=1}^{m} L_{k}\left(\delta_{k}-\varepsilon_{k}\right) \\
= & \frac{1+e^{\frac{1}{2}}}{2\left(\frac{1}{2}\right)\left(e^{\frac{1}{2}}-1\right)}\left[\int_{0}^{1}\left[\frac{1}{3} \int_{0}^{s} s^{2} r^{4} d r+\frac{1}{4} \int_{0}^{1} s^{3} r^{2} d r\right] d s+\frac{1}{3}\left(\frac{2}{5}-\frac{1}{5}\right)\right] \\
& +\left(\frac{1}{2}\right)\left(\frac{1}{2}\right)\left(\frac{1}{3}-\frac{1}{5}\right) \\
\approx & 0.4246196990<1,
\end{aligned}
$$




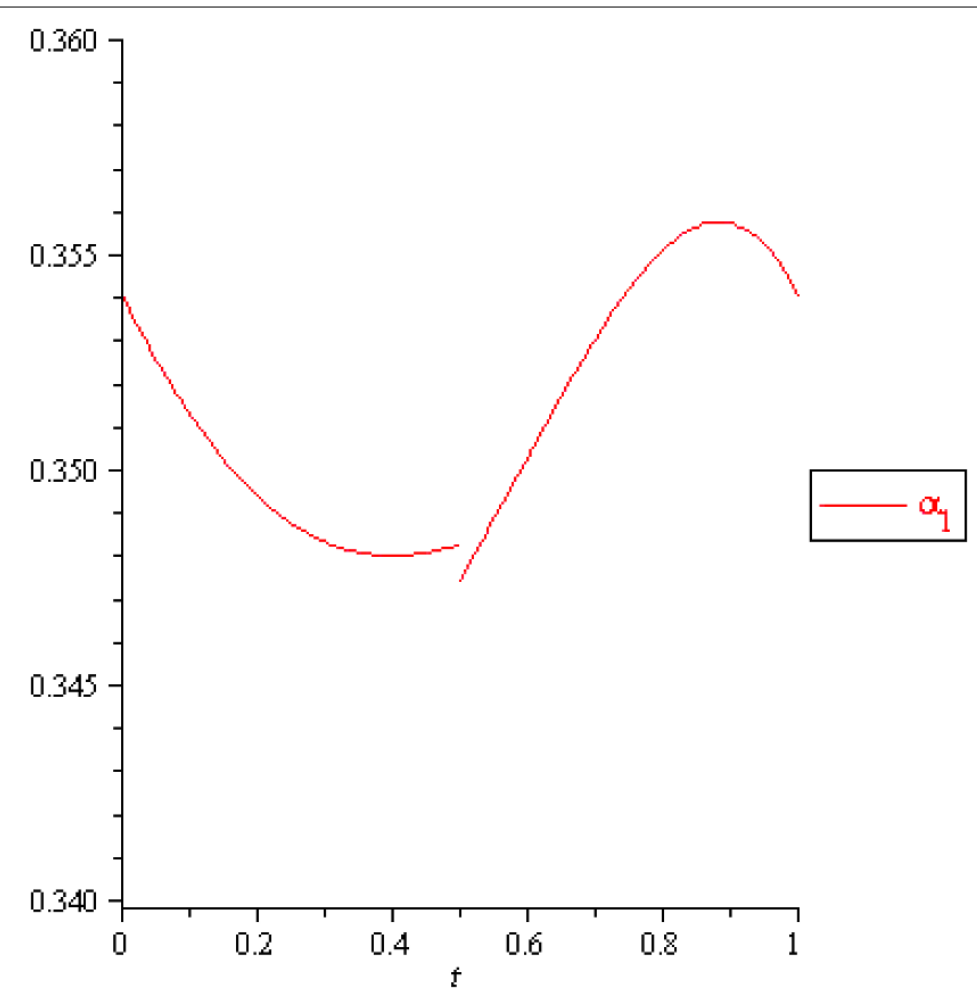

Figure 1 Time history of $\alpha_{1}$.

$$
\begin{aligned}
\mu= & \frac{1}{2}\left[\int_{0}^{T}\left[N \int_{0}^{s} k(s, r) d r+L \int_{0}^{T} h(s, r) d r\right] d s+\sum_{k=1}^{m} L_{k}^{*}\left(\tau_{k}-\sigma_{k}\right)\right] \\
& +\frac{\sqrt{M}\left(1+e^{\sqrt{M} T}\right)}{2\left(e^{\sqrt{M} T}-1\right)} \sum_{k=1}^{m} L_{k}\left(\delta_{k}-\varepsilon_{k}\right) \\
= & \frac{1}{2}\left[\int_{0}^{1}\left[\frac{1}{3} \int_{0}^{s} s^{2} r^{4} d r+\frac{1}{4} \int_{0}^{1} s^{3} r^{2} d r\right] d s+\frac{1}{3}\left(\frac{2}{5}-\frac{1}{5}\right)\right] \\
& +\frac{\frac{1}{2}\left(1+e^{\frac{1}{2}}\right)}{2\left(e^{\frac{1}{2}}-1\right)}\left(\frac{1}{2}\right)\left(\frac{1}{3}-\frac{1}{5}\right) \\
\approx & 0.1159664694<1 .
\end{aligned}
$$

Therefore, (4.1) satisfies all the conditions of Theorem 3.1. So, PBVP (4.1) has minimal and maximal solutions in the segment $\left[\alpha_{0}, \beta_{0}\right]$.

Substituting $\alpha_{0}, \beta_{0}$ into monotone iterative scheme, we obtain

$$
\left\{\begin{array}{l}
\alpha_{1}^{\prime \prime}(t)-\frac{1}{4} \alpha_{1}(t)-\frac{1}{3} \int_{0}^{t} t^{2} s^{4} \alpha_{1}(s) d s-\frac{1}{4} \int_{0}^{1} t^{3} s^{2} \alpha_{1}(s) d s \\
=-\frac{1}{2} t^{3}, \quad J=[0,1], t \neq \frac{1}{2}, \\
\Delta \alpha_{1}\left(\frac{1}{2}\right)=\frac{1}{2} \int_{\frac{1}{6}}^{\frac{3}{10}} \alpha_{1}^{\prime}(s) d s, \quad k=1, \\
\Delta \alpha_{1}^{\prime}\left(\frac{1}{2}\right)=\frac{1}{3} \int_{\frac{1}{10}}^{\frac{3}{10}} \alpha_{1}(s) d s, \quad k=1, \\
\alpha_{1}(0)=\alpha_{1}(T), \quad \alpha_{1}^{\prime}(0)=\alpha_{1}^{\prime}(T),
\end{array}\right.
$$




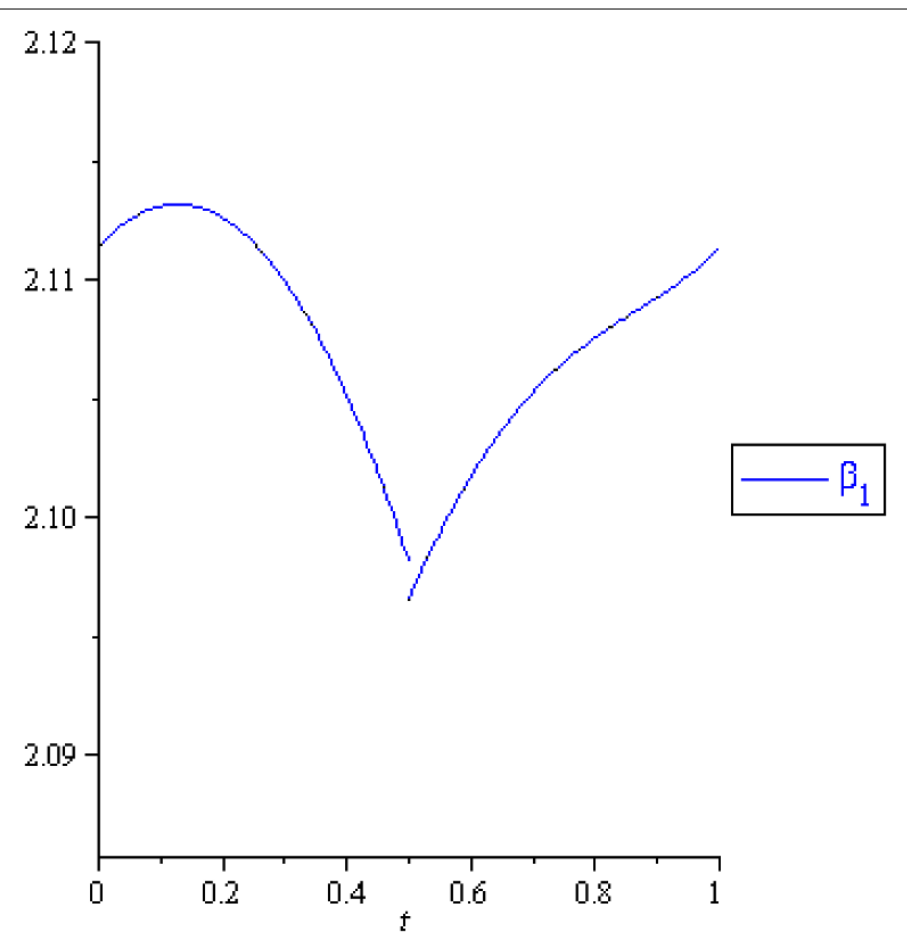

Figure 2 Time history of $\beta_{1}$.

and

$$
\left\{\begin{array}{l}
\beta_{1}^{\prime \prime}(t)-\frac{1}{4} \beta_{1}(t)-\frac{1}{3} \int_{0}^{t} t^{2} s^{4} \beta_{1}(s) d s-\frac{1}{4} \int_{0}^{1} t^{3} s^{2} \beta_{1}(s) d s \\
\quad=\frac{1}{10} t^{14}-\frac{1}{5} t^{7}+\frac{1}{8} t^{6}-\frac{3}{4}, \quad J=[0,1], t \neq \frac{1}{2} \\
\Delta \beta_{1}\left(\frac{1}{2}\right)=\frac{1}{2} \int_{\frac{1}{6}}^{\frac{3}{10}} \beta_{1}^{\prime}(s) d s, \quad k=1, \\
\Delta \beta_{1}^{\prime}\left(\frac{1}{2}\right)=\frac{1}{3} \int_{\frac{1}{10}}^{\frac{3}{10}} \beta_{1}(s) d s, \quad k=1, \\
\beta_{1}(0)=\beta_{1}(T), \quad \beta_{1}^{\prime}(0)=\beta_{1}^{\prime}(T) .
\end{array}\right.
$$

After using the variational iteration method [45] for (4.2), (4.3), the approximate solutions for $\alpha_{1}$ and $\beta_{1}$ can be illustrated as Figure 1 and Figure 2, respectively.

\section{Competing interests}

The authors declare that they have no competing interests.

\section{Authors' contributions}

All authors contributed equally in this article. They read and approved the final manuscript.

\section{Author details}

'Department of Mathematics, Faculty of Science, King Mongkut's Institute of Technology Ladkrabang, Bangkok, 10520, Thailand. ${ }^{2}$ Centre of Excellence in Mathematics, CHE, Sri Ayutthaya Road, Bangkok, 10400, Thailand. ${ }^{3}$ Department of Mathematics, Faculty of Applied Science, King Mongkut's University of Technology North Bangkok, Bangkok, 10800,

Thailand.

\section{Acknowledgements}

This research is supported by the Centre of Excellence in Mathematics, the Commission on Higher Education, Thailand. 


\section{References}

1. Bainov, DD, Simeonov, PS: Impulsive Differential Equations: Periodic Solutions and Applications. Longman, Harlow (1993)

2. Bainov, DD, Simeonov, PS: Impulsive Differential Equations: Asymptotic Properties of the Solutions. World Scientific, Singapore (1995)

3. Benchohra, M, Henderson, J, Ntouyas, S: Impulsive Differential Equations and Inclusions. Hindawi Publishing, New York (2006)

4. Lakshmikantham, V, Bainov, DD, Simeonov, PS: Theory of Impulsive Differential Equations. World Scientific, Singapore (1989)

5. Samoilenko, AM, Perestyuk, NA: Impulsive Differential Equations. World Scientific, Singapore (1995)

6. Ahmad, B, Alsaedi, A: Existence of solutions for anti-periodic boundary value problems of nonlinear impulsive functional integro-differential equations of mixed type. Nonlinear Anal. Hybrid Syst. 3, 501-509 (2009)

7. Boucherif, A, Fiagbedzi, YA: Periodic solutions of nonlinear integrodifferential equations. Dyn. Contin. Discrete Impuls. Syst. Ser. A, Math. Anal. 14, 165-175 (2007)

8. Chen, J, Tisdell, CC, Yuan, R: On the solvability of periodic boundary value problems with impulse. J. Math. Anal. Appl. 331, 902-912 (2007)

9. Ding, W, Mi, J, Han, M: Periodic boundary value problems for the first order impulsive functional differential equations. Appl. Math. Comput. 165, 433-446 (2005)

10. He, Z, Ge, W: Periodic boundary value problem for first order impulsive delay differential equations. Appl. Math. Comput. 104, 51-63 (1999)

11. He, Z, He, X: Monotone iterative technique for impulsive integro-differential equations with periodic boundary conditions. Comput. Math. Appl. 48, 73-84 (2004)

12. He, Z, He, X: Periodic boundary value problems for first order impulsive integro-differential equations of mixed type. J. Math. Anal. Appl. 296, 8-20 (2004)

13. He, Z, Yu, J: Periodic boundary value problem for first-order impulsive functional differential equations. J. Comput. Appl. Math. 138, 205-217 (2002)

14. He, Z, Yu, J: Periodic boundary value problem for first-order impulsive ordinary differential equations. J. Math. Anal. Appl. 272, 67-78 (2002)

15. Huseynov, A: Positive solutions of a nonlinear impulsive equation with periodic boundary conditions. Appl. Math Comput. 217, 247-259 (2010)

16. Li, J, Nieto, JJ, Shen, J: Impulsive periodic boundary value problems of first-order differential equations. J. Math. Anal. Appl. 325, 226-236 (2007)

17. Li, J, Shen, J: Periodic boundary value problems for delay differential equations with impulses. J. Comput. Appl. Math. 193, 563-573 (2006)

18. Li, J, Shen, J: Periodic boundary value problems for impulsive integro-differential equations of mixed type. Appl. Math. Comput. 183, 890-902 (2006)

19. Liang, $\mathrm{R}$, Shen, J: Periodic boundary value problem for the first order impulsive functional differential equations. J. Comput. Appl. Math. 202, 498-510 (2007)

20. Liu, Y: Further results on periodic boundary value problems for nonlinear first order impulsive functional differential equations. J. Math. Anal. Appl. 327, 435-452 (2007)

21. Liu, Y: Periodic boundary value problems for first order functional differential equations with impulse. J. Comput. Appl. Math. 223, 27-39 (2009)

22. Luo, Z, Jing, Z: Periodic boundary value problem for first-order impulsive functional differential equations. Comput. Math. Appl. 55, 2094-2107 (2008)

23. Nieto, Jj: Periodic boundary value problems for first-order impulsive ordinary differential equations. Nonlinear Anal. 51, 1223-1232 (2002)

24. Nieto, JJ, Rodríguez-López, R: Remarks on periodic boundary value problems for functional differential equations. J. Comput. Appl. Math. 158, 339-353 (2003)

25. Nieto, JJ, Rodríguez-López, R: Periodic boundary value problem for non-Lipschitzian impulsive functional differential equations. J. Math. Anal. Appl. 318, 593-610 (2006)

26. Tian, Y, Jiang, D, Ge, W: Multiple positive solutions of periodic boundary value problems for second order impulsive differential equations. Appl. Math. Comput. 200, 123-132 (2008)

27. Zhang, D, Dai, B: Infinitely many solutions for a class of nonlinear impulsive differential equations with periodic boundary conditions. Comput. Math. Appl. 61, 3153-3160 (2011)

28. Zhang, N, Dai, B, Qian, X: Periodic solutions for a class of higher-dimension functional differential equations with impulses. Nonlinear Anal. 68, 629-638 (2008)

29. Zhao, A, Bai, Z: Existence of solutions to first-order impulsive periodic boundary value problems. Nonlinear Anal. 71 1970-1977 (2009)

30. Han, J, Liu, Y, Zhao, J: Integral boundary value problems for first order nonlinear impulsive functional integro-differential equations. Appl. Math. Comput. 218, 5002-5009 (2012)

31. Hao, X, Liu, L, Wu, Y: Positive solutions for second order impulsive differential equations with integral boundary conditions. Commun. Nonlinear Sci. Numer. Simul. 16, 101-111 (2011)

32. Jankowski, T: Positive solutions for second order impulsive differential equations involving Stieltjes integral conditions. Nonlinear Anal. 74, 3775-3785 (2011)

33. Song, $G$, Zhao, $Y$, Sun, $X$ : Integral boundary value problems for first order impulsive integro-differential equations of mixed type. J. Comput. Appl. Math. 235, 2928-2935 (2011)

34. Wang, G, Zhang, L, Song, G: New existence results and comparison principles for impulsive integral boundary value problem with lower and upper solutions in reversed order. Adv. Differ. Equ. 2011, Article ID 783726 (2011)

35. Zhang, X, Feng, M, Ge, W: Existence of solutions of boundary value problems with integral boundary conditions for second-order impulsive integro-differential equations in Banach spaces. J. Comput. Appl. Math. 233, 1915-1926 (2010)

36. Ding, W, Han, M: Periodic boundary value problem for the second order impulsive functional differential equations. Appl. Math. Comput. 155, 709-726 (2004) 
37. Ding, W, Han, M, Mi, J: Periodic boundary value problem for the second-order impulsive functional differential equations. Comput. Math. Appl. 50, 491-507 (2005)

38. Li, J: Periodic boundary value problems for second-order impulsive integro-differential equations. Appl. Math Comput. 198, 317-325 (2008)

39. Liang, R, Shen, J: Periodic boundary value problem for second-order impulsive functional differential equations. Appl. Math. Comput. 193, 560-571 (2007)

40. Yang, $X$, Shen, J: Periodic boundary value problems for second-order impulsive integro-differential equations. J. Comput. Appl. Math. 209, 176-186 (2007)

41. Yao, M, Zhao, A, Yan, J: Periodic boundary value problems of second-order impulsive differential equations. Nonlinear Anal. 70, 262-273 (2009)

42. Nieto, JJ: An abstract monotone iterative technique. Nonlinear Anal., Theory Methods Appl. 28, 1923-1933 (1997)

43. Tariboon, J: Boundary value problems for first order functional differential equations with impulsive integral conditions. J. Comput. Appl. Math. 234, 2411-2419 (2010)

44. Liu, Z, Han, J, Fang, L: Integral boundary value problems for first order integro-differential equations with impulsive integral conditions. Comput. Math. Appl. 61, 3035-3043 (2011)

45. Wazwaz, AM: Linear and Nonlinear Integral Equations: Methods and Applications. Springer, New York (2011)

doi:10.1186/1687-2770-2012-122

Cite this article as: Thaiprayoon et al.: Periodic boundary value problems for second-order impulsive integro-differential equations with integral jump conditions. Boundary Value Problems 2012 2012:122.

\section{Submit your manuscript to a SpringerOpen ${ }^{\ominus}$ journal and benefit from:}

- Convenient online submission

- Rigorous peer review

Immediate publication on acceptance

- Open access: articles freely available online

- High visibility within the field

- Retaining the copyright to your article 